\title{
Probabilistic Inference Approach for Predicting Concrete Compressive Strength - A Bayesian Network Algorithm
}

\author{
Omar Najm', Hilal El-Hassan', Amr El-Dieb ${ }^{1}$, Hamad Aljassmi ${ }^{1}$ \\ ${ }^{1}$ United Arab Emirates University \\ P.O. Box 15551, Al Ain, UAE \\ 201890004@uaeu.ac.ae; helhassan@uaeu.ac.ae; amr.eldieb@uaeu.ac.ae; h.aljasmi@uaeu.ac.ae
}

\begin{abstract}
This study highlights innovative and novel techniques that employ Artificial Intelligence (AI) technology in evaluating and predicting concrete compressive strength. Past literature utilized different AI algorithms to predict the nonlinear behaviour of concrete, of which the most commonly used is the Artificial Neural Network (ANN). Limited past studies used the probabilistic inference approach by using Bayesian Networks (BN) to envisage the structural health integrity and mechanical performance of concrete. This research investigates the potential applicability of $\mathrm{BN}$ in predicting the compressive strength of self-compacting concrete made with various supplementary cementitious materials and basalt fibers. Two learning algorithms, namely Naïve Bayes and Markov Blanket, were employed along with various discretization methods to maximize network performance and minimize integral absolute error. Research findings showed that Naïve Bayes classifier, coupled with K-means discretization tool with 4 segments of 'days' variable and 3 segments of the remaining variables, gave the highest correlation between experimental and predicted values. The accuracy of the predicted BN results was slightly superior to that obtained from the ANN model.
\end{abstract}

Keywords: Probabilistic Inference, Bayesian Network, Artificial Neural Network, Compressive Strength, Sustainable Concrete.

\section{Introduction}

Bayesian network is a graphical representation of probabilistic models that shows causal and effect relations between the variables and deals with uncertainties of a domain $[1,2]$. It follows the concept of the probability theory, by which a certain domain is defined with a set of random variables of possible occurrences. The visual illustration of the network represents the conditional dependencies among variables for a given problem of this domain [2,3]. It shows the effect of any two conditionally independent variables on each other through intermediary variables that separate them [3]. The proposed mathematical equation used in BN algorithms is expressed in Eq. (1).

$$
\mathrm{P}(\mathrm{H} \mid \mathrm{E}, \mathrm{c})=\frac{\mathrm{P}(\mathrm{H} \mid \mathrm{c}) \times \mathrm{P}(\mathrm{E} \mid \mathrm{H}, \mathrm{c})}{\mathrm{P}(\mathrm{E} \mid \mathrm{c})}
$$

In Eq. (1), H, E, and c represent the hypothesis, evidence, and background context, respectively. The term $\mathrm{P}(\mathrm{H} \mid \mathrm{E}, \mathrm{c})$ denotes the posterior probability of the hypothesis after considering the effect of the evidence $\mathrm{E}$ on the context $\mathrm{c}$. The term $\mathrm{P}(\mathrm{H} \mid \mathrm{c})$ represents the prior probability of $\mathrm{H}$ with respect to $\mathrm{c}$ alone, while $\mathrm{P}(\mathrm{E} \mid \mathrm{H}, \mathrm{c})$ indicates the likelihood of having the evidence in the case of the hypothesis and the context both true. The last term, $\mathrm{P}(\mathrm{E} \mid \mathrm{c})$, can be considered as normalizing or scaling factor, as it is independent of $\mathrm{H}$ [4]. The posterior probability distribution of $\mathrm{BN}$ is obtained by multiplying the likelihood "L", which is defined by Eq. (2), with the prior probability and then normalizing.

$$
\mathrm{P}(\mathrm{E} \mid \mathrm{H}, \mathrm{c})=\mathrm{L}(\mathrm{H})=\prod_{\mathrm{i}} \mathrm{P}\left(\mathrm{E}_{\mathrm{i}} \mid \mathrm{H}\right)
$$

Bayesian networks are built on two main pillars, qualitative and quantitative models [5]. For the qualitative models, BN correlates and finds the relationship among the variables through a causal structure learning approach [6]. Then, a direct 
acyclic graph (DAG) is generated to represent the variables of interest through the nodes and the casual dependencies through the directed links connecting the nodes. For instance, a simple BN structure for producing a research publication is shown in Fig. 1. In this aspect, the arrow links are directed from the parent node to its child node. As the network adopts an acyclic approach, the possibility of ending up reaching the same starting node is non-existent [7]. On the other hand, the quantitative models mainly concern the local probability distribution for specifying the probabilistic relationships by which the dependencies are quantified. This is performed by conditional probability tables (CPT) that classify nodes with their parents [5].

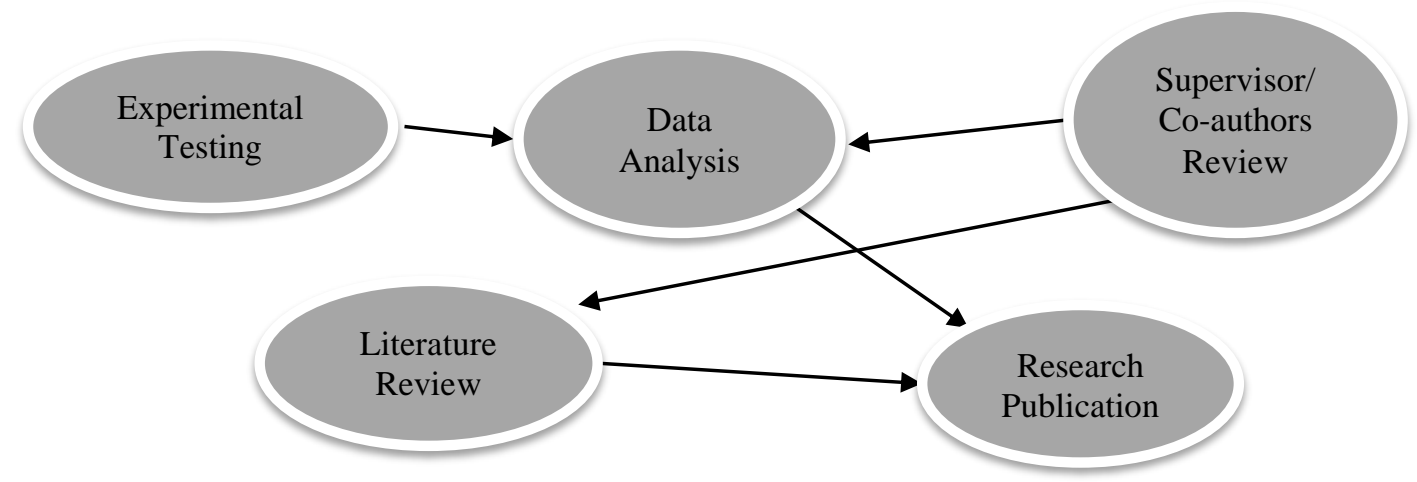

Fig. 1: Simple Bayesian network structure of a research publication.

Bayesian networks have been implemented in a wide range of real-world applications due to their ease in the estimation of certain variables, treatment of uncertainties, and decision analysis with quick responses to the user [2]. The major applications that extensively utilize $\mathrm{BN}$ are risk management, quality management, financial analysis, medicine applications and diagnosis, psychological applications, and various engineering applications [2, 8]. Despite the capability of experimenting with both continuous and discrete variables, many Bayesian network algorithms only deal with the latter. As such, all continuous variables are discretized and then classified into different categories (states), such as high, medium, and low [7].

The probabilistic inference of the network is based on four steps, initial probability distribution, reasoning from cause to effect, combined influence of variables, and reasoning from the effect to the cause [7]. Further, BN learning algorithms are highly dependent on the type of infused data. In the event of using data without a hypothesis constraining the relationships between the variables, the unsupervised learning algorithms are employed. Otherwise, the supervised learning algorithms such as Naïve Bayes and Markov Blanket can be used for the inference. Naïve Bayes is considered one of the most effective learning algorithms for supervised machine learning, as it is based on the independencies between the predictors [7,9]. On the other hand, Markov Blanket algorithm is usually used for high-dimensional models where too many variables affect the target node [10].

Based on the available literature, the use of the probabilistic inference approach to anticipate sustainable selfcompacting concrete performance has not been investigated yet. Thus, the aim of this study is to evaluate the ability to employ Bayesian network algorithms to predict the mechanical properties of sustainable self-compacting concrete by which a large amount of cement content was replaced with different supplementary by-products materials. The probability-based network results are compared with those of artificial neural networks that are obtained from the same dataset. It is anticipated that the probability approach can lead to a more in-depth understanding of the interaction between the concrete ingredients and their effect on the final concrete properties, while also providing a numerical platform for performance prediction of sustainable self-compacting concrete. 


\section{Experimental Methodology}

The dataset, obtained from past literature [11], is characterized by the constituents and compressive strength results of of high strength self-compacting concrete (SCC). To enhance the sustainability of the concrete, mixes were designed to attain $80 \%$ cement replacement with different combinations of supplementary cementitious materials (SCM). The supplementary cementitious materials used in the study were fly ash, silica fume, and ground granulated blast furnace slag slag (GGBS). The cement replacement strategy was carried out through binary, ternary, and quaternary mixes at different percentages ratios. For the binary mixes, cement was replaced with one SCM at a time. The replacement range for each SCM was chosen based on recommendations from past literature [12-16]. Accordingly, fly ash, silica fume, and GGBS replaced cement by 0-40, 0-20, and 0-80\%. In ternary mixes, the combined effect of two SCM percentages as partial cement replacement was studied. The quaternary mixes were composed of all three SCMs in different combinations to replace $80 \%$ of cement content. For all generated mixes, four different basalt fibers were used, ranging from 0.25 to $1 \%$ by volume at $0.25 \%$ increments.

To minimize the number of variables in the study and focus on the effect of SCMs and basalt fibers, all other mixture proportioning variables were kept fixed. For instance, water-to-binder ratio was set to 0.36 and the flowability of concrete was maintained between 400 and $700 \mathrm{~mm}$ through the use of a high-range water reducer, HRWR (1.5\%, by binder mass). Fine and coarse aggregates were also set to 896 and $800 \mathrm{~kg} / \mathrm{m}^{3}$, respectively. The compressive strength was measured after 3,7 , and 28 days of water curing at room temperature. Experimental test results comprised 226 data points that varied based on SCM content, basalt fibers content, testing age, and compressive strength. All other fixed constituents were removed from the data, as they had no effect on the training cycles. The developed data set was used to train Bayesian Network (BN) with a commercial tool called Bayesialab using two learning algorithms, Naïve Bayes and Markov Blanket. The results obtained from the BN model were then compared to predicted results acquired from a self-developed code of Artificial Neural Network (ANN).

\subsection{Bayesian Network}

The Bayesian Network prediction model was developed through the use of Bayesialab commercial software. Before processing the data set into the software, it is essential to know the domain and set of variables affecting the results [7]. Such knowledge will ease the construction of DAG for efficient training and prediction cycles. The accuracy of the prediction algorithm was also improved by choosing the right discretization method to convert the continuous data into discrete ones. Regardless of the network structure and discretization method, the selected machine learning algorithm has the highest impact on the inference of the network; thus it was essential to examine many algorithms to enhance the accuracy of the results. In the current study, a model is developed to relate multiple independent variables, such as the type and content of the binding materials, water to binder ratio, additives, type and quantity of coarse and fine aggregates, and testing age, to the concrete compressive strength. As the content of fine and coarse aggregates and the HRWR additive were fixed to a certain amount, they were omitted during the network modeling preparation. As such, the used variables were compressive strength (CS), cement (C), fly ash (F), silica fume (S), GGBS (G), basalt fibers (B), and testing age in days (D)). The probability of having certain compressive strength given the observations of the other variables is proposed in the following Eq. (3):

$$
\mathrm{P}(\mathrm{C} \mid \mathrm{C}, \mathrm{F}, \mathrm{S}, \mathrm{G}, \mathrm{B}, \mathrm{D})=\frac{\mathrm{P}(\mathrm{CS}, \mathrm{C}, \mathrm{F}, \mathrm{S}, \mathrm{G}, \mathrm{B}, \mathrm{D})}{\mathrm{P}(\mathrm{C}, \mathrm{F}, \mathrm{S}, \mathrm{G}, \mathrm{B}, \mathrm{D})}
$$

The datasets were organizing in a table of seven columns, whereby the first six columns represented the concrete constituents (cement, fly ash, silica fume, GGBS, basalt fibers, and days) and the last column showed the corresponding compressive strength result. In total, 226 data points, representing 75 different mixes with 3 different testing ages, were inputted. Further, the data was processed by subdividing it into training and testing sets. This process is important to validate the strength of the prediction model and increase its accuracy. In fact, $20 \%$ of data are typically used for the testing data, and the remaining $80 \%$ is used for the learning algorithm. 


\subsection{Artificial Neural Network}

An artificial neural network (ANN) was used to train and predict the same concrete data set employed in the network prediction tool. Back-propagation ANN code was developed with MATLAB coding interface and used to train test the data. The developed code was for a one-hidden layer network that used the log-sigmoid transfer function to the output results and the sum of square error (SSE) with a minimum value of 0.1 to stop the training process. The initial weights of the input variables were chosen randomly with values between -0.5 and 0.5 , while the learning rate responsible for changing the weights after each learning cycle was used in two values, 0.05 and 0.1 . Similar to BN, the ANN training and testing data were $80 \%$ and $20 \%$ from the original data set, respectively. Actually, the exact same training and testing data were employed in ANN, as it serves as a benchmark to verify the accuracy of the BN inference. The ideal ANN configuration was determined through analyzing and testing 14 different architectures, whose optimum was established based on the correlation coefficient, $\mathrm{R}^{2}$, between the evaluated results and testing data. The main parameters to be changed were the learning rate and the number of neurons. As a result of this process, the ANN employed in the study was with 6 inputs values and 28 neurons with 1 output at a learning rate of 0.05 (6-28-1 architecture).

\section{Results and Discussion}

\subsection{Bayesian Network}

The statistical approach of finding concrete compressive strength was highly dependent on the training and testing data and the discretization method employed. Using Naïve Bayes and Markov Blanket algorithms, the optimum discretization method of the network was determined by measuring integral absolute error (IAE, \%) shown in Eq. (4), as it is statically more sensitive than ordinary average error [17].

$$
\operatorname{IAE}(\%)=\sum \frac{\left[(\mathrm{Ei}-\mathrm{Pi})^{2}\right]^{0.5}}{\sum \mathrm{Ei}} \times 100
$$

Where $E_{i}$ and $P_{i}$ are the experimental and predicted results.

Table 1: Different discretization tools used in the study.

\begin{tabular}{|l|l|l|}
\hline Discretization Tool & IAE\% & Comments \\
\hline R2-GenOpt & 8.8 & Default \\
\hline R2-GenOpt & 6.2 & 3 days intervals \\
\hline Density Approximation & 11.3 & 3 intervals \\
\hline Density Approximation & 11.3 & 5 intervals \\
\hline K-Means & 8.27 & Default 3 intervals \\
\hline K-Means & 4.26 & 4 days intervals \\
\hline K-Means & 7.37 & 4 intervals all \\
\hline Equal Distances & 11.1 & Default 3 intervals \\
\hline Equal Distances & 13.9 & 7 intervals \\
\hline Equal Frequencies & 9.2 & Default 3 intervals \\
\hline Equal Frequencies & 9.9 & 7 intervals \\
\hline
\end{tabular}

For the Naïve Bayes approach, five different discretization algorithms were tested to maximize the network's performance and to reduce the IAE below 10\%, as to be acceptable [17]. Table 1 summarizes the IAE of the employed methods. It was found that the k-means method with 4 segments of the 'days' variable and 3 segments of the remaining 
variables gave the highest correlation between all other methods with IAE\% of $4.26 \%$. The $\mathrm{R}^{2}$ of the trained data is found to be 0.71 , meanwhile, the predicted compressive strength of the testing set gave higher accuracy with $\mathrm{R}^{2}$ of 0.91 . The correlation between the predicted and the experimental values of compressive strength for the training and testing dataset is illustrated in Fig. 2(a) and (b), respectively.

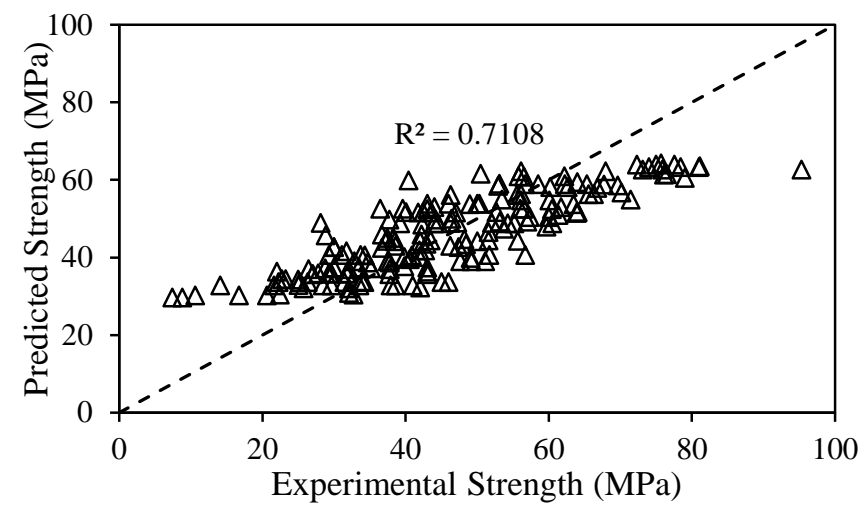

(a)

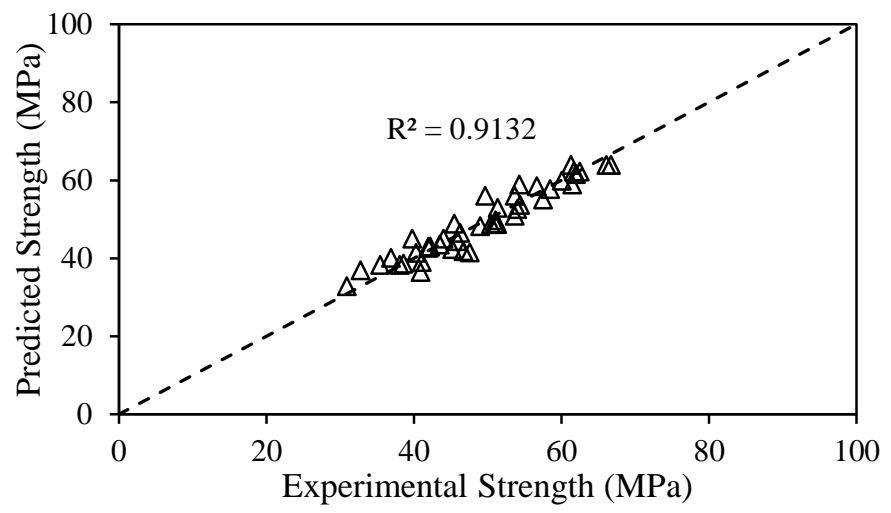

(b)

Fig. 2: Naïve Bayes approach for correlating (a) BN training and experimental strength results and (b) BN testing and experimental strength results.

As shown in Fig. 2(a), the correlation coefficient, $\mathrm{R}^{2}$, between the trained and predicted data was 0.71 . Besides this relatively imprecise correlation, it is clear that the predicted compressive strength results are deviated from the line of equality at values below $20 \mathrm{MPa}$ and above $60 \mathrm{MPa}$. In comparison, $\mathrm{R}^{2}$ between the testing and predicted data was 0.91 , as illustrated in Fig. 2(b), with a more accurate prediction of the testing data.

For Markov Blanket learning algorithm, it only used the dataset of 'days' relating to the testing age to predict the concrete compressive strength. The nodes connecting to other variables were removed. The overall precision of the learning and testing data sets was $60 \%$ and 53\%, respectively. The predicted values of compressive strength had IAE\% of $13.8 \%$ and $\mathrm{R}^{2}$ of 0.46 , as seen in Fig. 3. In fact, scatter plot results show that only three compressive strength values were predicted, namely 34,44 , and $58 \mathrm{MPa}$. Such low precision and inaccuracy can be attributed to the limiting action of the MB algorithm to the required nodes for analysis and to restricting the variables to the testing age only.

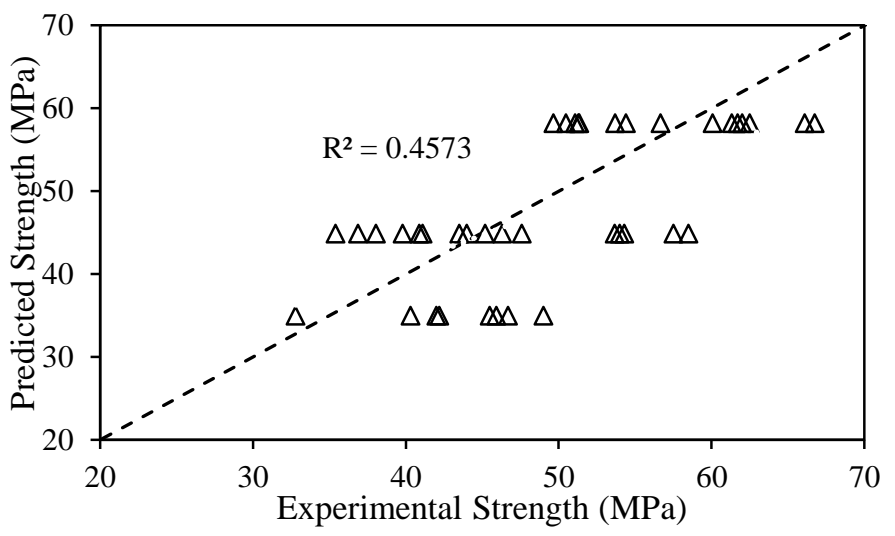

Fig. 3: Markov Blanket approach for correlating predicted and experimental strength results.

\subsection{Bayesian Network vs. Artificial Neural Network}


The correlation coefficients of Bayesian network training and testing phases by Naïve Bayes classifier were 0.71 and 0.91 , respectively. The prediction sensitivity and accuracy were found to be related to the discretization method used before processing the data. Additionally, the use of different discretization tools within the same model gave more accurate results. In contrast, outcomes of the ANN for training and testing phases were in high agreement with the target results with $\mathrm{R}^{2}$ of 0.95 and 0.90 , respectively. Its integral absolute error was $4.27 \%$. The accuracy of the ANN can be attributed to the absence of a data discretization method before processing, as the learning algorithm depends on finding the error between the predicted and target values, distributing the differences among all the initial weights, and continuing the process until a high level of accuracy is attained.

Fig. 4(a) illustrates the compressive strength results obtained from experimental testing and those predicted by BN and ANN. It is clear that both models converge close to the $45^{\circ}$-line, indicating a high level of accuracy in predicting the compressive strength using BN or ANN. Further, to evaluate the performance of the developed algorithms, the error between the experimental and predicted strength is plotted in Fig. 4(b). The range of error generally varies between -10 and $+10 \%$, with only 7 out of the 90 data points $(7.8 \%)$ being outside this range. The trend lines associated to the ANN and $\mathrm{BN}$ prediction data points are also plotted. The respective slopes of the two networks, 0.061 and 0.013 , indicate that the Bayesian network structure renders a slightly more accurate and precise prediction of the compressive strength.

The predicted strength results are also analyzed using hypothesis testing with a 95\% confidence interval $(\alpha=0.05)$. The null hypotheses tested consider that the strength predicted by each of BN and ANN are not different than those obtained from the experimental testing. The alternative hypotheses are that the predicted and experimental results are different. Results show that the respective t-values of BN and ANN testing are 0.0049 and 0.0313, which are less than 1.98. Thus, the null hypothesis cannot be rejected. As such, the strength values acquired from BN and ANN are not different than those attained from experimental testing, i.e., both structures could be used in predicting the concrete compressive strength. Yet, it seems that BN is slightly more accurate, which is agreement with the error analysis.

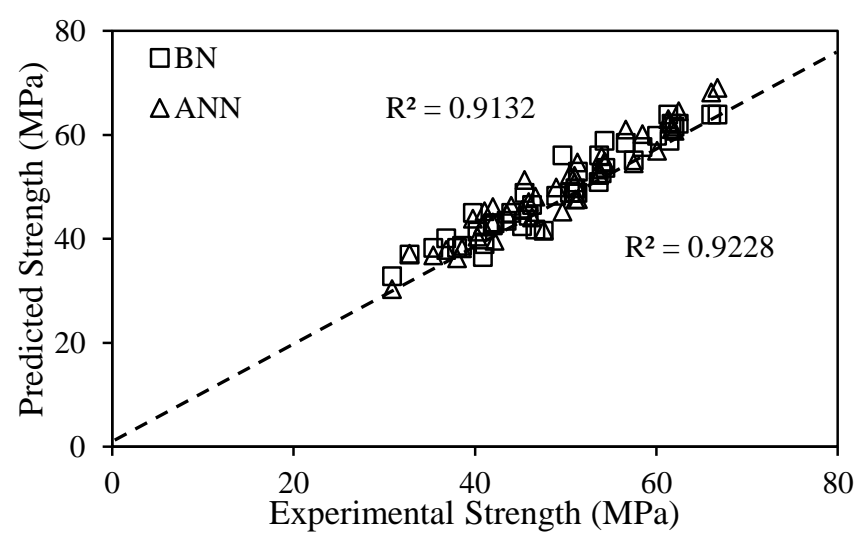

(a)

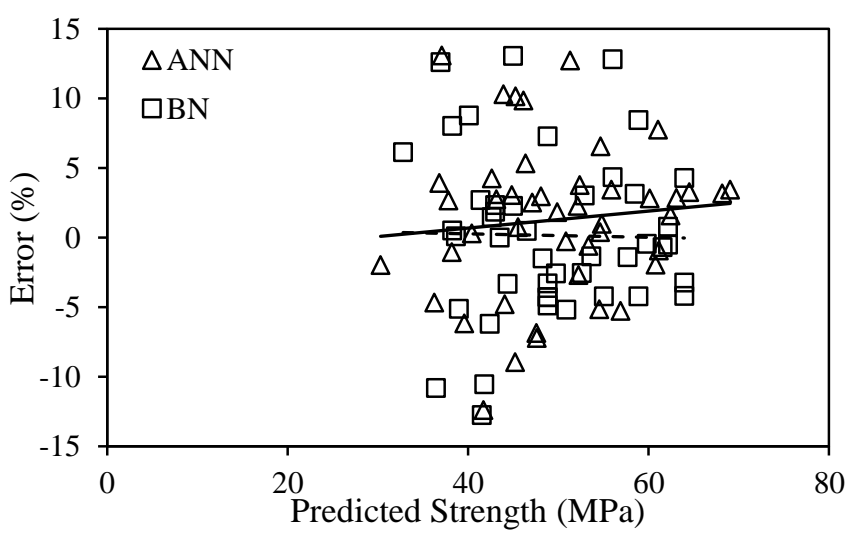

(b)

Fig. 4: (a) Correlation among experimental, BN and ANN results and (b) the error attributed to the ANN and BN predicted strength results.

\section{Conclusions}

The use of a probability-based algorithm is a novel approach for predicting the mechanical performance of concrete. The Bayesian Network structure was implemented with different discretization techniques to provide a numerical platform for predicting the compressive strength of self-compacting concrete made with supplementary cementitious materials. The results showed that Naïve Bayes algorithm, along with the K-mean model discretization of 4 intervals of 'days' dataset and 3 intervals for the remaining ones, led to a high level of inference with IAE and $\mathrm{R}^{2}$ of $4.26 \%$ and 0.91, respectively. Markov Blanket algorithm failed to predict the behavior of the compressive strength with its dependence on the 'days' parameter solely. The accuracy and precision of the predicted Bayesian network were slightly superior to that obtained from an ANN model. The novelty of this work resides in the ability to employ probabilistic 
approaches using Bayesian Networks to effectively predict concrete compressive strength rather than conducting costly, labor-intensive, and time-consuming laboratory testing. Future work may explore the applicability of the BN approach in in envisaging the mechanical and long-term durability performance of different types of concrete.

\section{Acknowledgements}

The authors gratefully acknowledge the financial support of UAE University under grant number 31 N322.

\section{References}

[1] D. Heckerman, "A tutorial on learning with Bayesian networks," Microsoft Research, Redmond, Technical Report MSR-TR-95-06, 1996.

[2] L. Antonio Pereira Silva, J. Batista Nunes Bezerra, M. Barbosa Perkusich, K. Costa Gorgônio, H. Oliveira de Almeida, and A. Perkusich, "Continuous learning of the structure of Bayesian networks: A Mapping Study," in Bayesian Networks - Advances and Novel Applications, D. McNair, Ed. IntechOpen, 2019, pp. 55-72.

[3] T. Stephenson, "An introduction to Bayesian network theory and usage," Dalle Molle Institute for Perceptual Artificial Intelligence, Martigny, Switzerland, Research Report IDIAP-RR 00-03, 2000.

[4] D. Niedermayer, "An introduction to Bayesian networks and their contemporary applications," in Innovations in Bayesian Networks: Theory and Applications, D. E. Holmes and L. C. Jain, Eds. Berlin, Heidelberg: Springer, 2008, pp. 117-130.

[5] I. Ben-Gal, "Bayesian networks," in Encyclopedia of Statistics in Quality and Reliability, American Cancer Society, 2008.

[6] J. Pearl, "Causal diagrams for empirical research," University of California, LA, USA, Technical Report R-218-B, 1995.

[7] V. A. Phan, "Towards global experimental design using Bayesian networks: cast studies on modeling sensory satiation," Ph.D Thesis, Wageningen University, Netherlands, 2013.

[8] N. Asakura and T. Inui, "A Bayesian framework for false belief reasoning in children: A Rational Integration of TheoryTheory and Simulation Theory," Front. Psychol., vol. 7, Dec. 2016, doi: 10.3389/fpsyg.2016.02019.

[9] P. H. Sydenham and R. Thorn, Eds., "129: Artificial neural networks," in Handbook of measuring system design, vol. 2, 3 vols., Chichester, England: Wiley, 2005.

[10] S. Fu and M. C. Desmarais, "Markov Blanket based feature selection: A review of past decade," in Proceedings of the World Congress on Engineering, London, UK, 2010, vol. 1, p. 8.

[11] O. A. Mohamed and O. F. Najm, "Compressive strength and stability of sustainable self-consolidating concrete containing fly ash, silica fume, and GGBS," Front. Struct. Civ. Eng., vol. 11, no. 4, pp. 406-411, 2017.

[12] P. Dinakar, M. Kartik Reddy, and M. Sharma, "Behaviour of self-compacting concrete using Portland pozzolana cement with different levels of fly ash," Mater. Des., vol. 46, pp. 60-616, 2013.

[13] ACI Committee 233, "Slag cement in concrete and mortar." American Concrete Institute, Farmington Hills, Michigan, 2003.

[14] N. Jin, "Fly ash applicability in pervious concrete," Master Thesis, The Ohio State University, 2010.

[15] H. El-Hassan and P. Kianmehr, "Pervious concrete pavement incorporating GGBS to alleviate pavement runoff and improve urban sustainability," Road Mater. Pavement Des., vol. 19, no. 1, pp. 167-181, 2018.

[16] C. Jaturapitakkul, K. Kiattikomol, V. Sata, and T. Leekeeratikul, "Use of ground coarse fly ash as a replacement of condensed silica fume in producing high-strength concrete," Cem. Concr. Res., vol. 34, no. 4, pp. 549-555, 2004, doi: 10.1016/S0008-8846(03)00150-9.

[17] F. A. Oluokun, E. G. Burdette, and J. H. Deatherage, "Splitting tensile strength and compressive strength relationships at early ages," ACI Mater. J., vol. 88, no. 2, pp. 115-121, 1991, doi: 10.14359/1859. 